\title{
ASPETTI GIURIDICI E POLITICI DELLA STERILIZZAZIONE
}

\author{
Massimo Paradiso \\ Professor Titular da Universidade de Catania
}

1. Per sterilizzazione s'intende propriamente l'atto con cui si provoca la sterilità in una persona sessualmente feconda. Possono distinguersi, fondamentalmente, due tipi di sterilizzazione: organica, che si ottiene tramite la modificazione $\mathrm{o}$ asportazione degli organi deputati alla procreazione, e funzionale, consistente nel blocco della funzionalità di detti organi tramite farmaci (per lo più, estro-progestinici sintetici). Quest'ultima è una sterilizzazione temporanea, e perciò di norma reversibile con la semplice sospensione del trattamento farmacologico; la prima, vice-versa, è destinata a durare nel tempo ed è anzi, di norma, irreversibile: è a quest'ultimo tipo di pratica medica che si fa comunemente riferimento quando si parla di sterilizzazione e che costituisce l'oggetto di queste note.

Ma ulteriori distinzioni attengono al piano degli scopi o risultati perseguiti. Si denomina terapeutica la sterilizzazione non direttamente voluta in quanto tale, bensì conseguente a un intervento necessitato dall'esigenza di cura della persona (ad es., l'asportazione dell'utero affetto da carcinoma). Va definita invece antiprocreativa (o, impropriamente, anticoncezionale) la sterilizzazione perseguita direttamente al fine di rendere la persona incapace di procreare; ne sono sottotipi la sterilizzazione su indicazione medica, diretta ad evitare gravidanze che siano fonte di pericolo per la vita della gestante; quella eugenetica, praticata al fine di evitare la trasmissione di un patrimonio genetico indesiderato (malattie o particolari caratteri ereditari); quella edonistica, o di comodo, determinata dal solo desiderio di evitare la procreazione. Si distingue infine, su un piano diverso, la sterilizzazione volontaria, richiesta dal singolo, da quella forzata o coatta, impostta cioè dall'esterno.

Si tratta di distinzioni ormai ben note, ma sulle quali conviene richiamare l'attenzione, sia perché tende ad accreditarsi qualche confusione in proposito, sia per dar conto della qualificazione antiprocreativa - preferita a quella corrente, di anticoncezionale - per le sterilizzazioni edonistica ed 
eugenetica. Invero, si nota la tendenza a ricomprendere in un generico calderone definito contraccezione sia mezzi in realtà abortivi, come la pillola del giomo dopo e la spirale, sia la stessa sterilizzazione definitiva. In realtà, si tratta di interventi che operano su fasi biologicamente distinte del processo generativo umano e che sarebbe del tutto arbitrario confondere. ${ }^{1}$

Per altro verso, preme qui evidenziare come la sterilizzazione ha bensì un generale significato antiprocreativo, derivandone comunque uno stabile impedimento alla procreazione; su esso tuttavia si innestano diversi significati specifici, in quanto diretta, di volta in volta, a rispondere a un problema che può essere individuale, di coppia, sociale o politico: dalla libertà personale in campo sessuale alla sterilità coniugale, dalla indicazione medica e terapeutica alla finalità eugenetica privata, dalla pianificazione demografica collettiva fino alla programmazione eugenetica e razziale di tragica memoria.

$\mathrm{Si}$ tratta dunque di funzioni diverse che, non di rado, vengono sovrapposte o cumulate e rischiano perciò di oscurare il reale significato concreto di quel che si definisce, genericamente, sterilizzazione. E' del tutto evidente, infatti, che le motivazioni individuali possono confliggere, o almeno non coincidere, con quelle sociali, che la sterilizzazione forzata non può che avere scopi e giustificazioni diverse da quella volontaria, che la pratica della limitazione delle nascite, che comunque ne consegue, non può che avere finalità diverse nel mondo industrializzato e nei paesi in via di sviluppo.

D'altra parte, è ben noto che la pratica della sterilizzazione ha ampia diffusione nel mondo e viene tollerata, quando non incoraggiata o addirittura imposta, sia da singoli paesi sia da organizzazioni internazionali. In tal senso, la sterilizzazione è un dato d'esperienza di cui occorre prendere atto per quello che è: una pratica diffusa che risponde a finalità diverse.

Mi sembra utile, allora, iniziare il discorso da un esame delle ragioni comunemente addotte a sostegno della liceità e desiderabilità della

1. Sinteticamente, in tale processo possono distinguersi le seguenti fasi: 1) elaborazione delle cellule germinali e 2) loro trasferimento all'interno dell'organismo maschile o femminile; 3) passaggio del seme maschile nelle vie genitali femminili interne e 4) concepimento; 5) gestazione; 6) parto. Ebbene, la sterilizzazione interviene sulle prime due fasi, incidendo sulla capacità di fecondare o di essere fecondata; la contraccezione, propriamente, opera sulla seconda e la terza, impedendole; l'aborto, infine, opera sulla penultima fase del processo generativo, interrompendo la gravidanza in corso. 
sterilizzazione volontaria come mezzo di controllo e pianificazione delle nascite. Ragioni che, schematicamente, si rifanno alla tutela della salute, alla garanzia della libertà individuale, alle esigenze di sviluppo economico, in particolare dei paesi cd. emergenti. Specifici rilievi saranno dedicati poi, di volta in volta, alla sterilizzazione forzata.

2. La prima delle motivazioni addotte a difesa della sterilizzazione, dunque, fa riferimento alla salvaguardia della salute. In particolare, si afferma che la sterilizzazione, pur menomando l'integrità física della persona, salvaguarda la salute complessivamente intesa perché soddisfa altre esigenze, come la libertà individuale nei rapporti sessuali, l'armonia di rapporti di coppia liberi dal timore della prole, la finalità di evitare la trasmissione di malattie ereditarie. In altre parole, la lesione dell'integrità fisica sarebbe compensata dal benessere psichico che ne consegue.

D'altra parte, la salute viene intesa non come semplice assenza di malattie, bensì nella sua accezione più ampia, e oggi largamente recepita, di completo stato di benessere sia fisico che psichico. Un concetto, si precisa, diverso e più ampio rispetto a quello di integrità fisica: questa infatti costituisce solo un pressuposto, necessario ma non sufficiente, della salute e, se del caso, potrà essere sacrificata per la salute dell'organismo nel suo complesso.

Su tali ultime precisazioni a parte qualche riserva sulla definizione di salute, che appare quanto meno velleitaria non si può non consentire, costituendo il principio terapeutico (e cioè il principio della sacrificabilità della parte per la salvaguardia del tutto) il fondamento stesso dell'attività medica e chirurgica. Viceversa, desta gravi perplessità il fatto che fra i pressupposti della salute entri soltanto l'integrità fisica e non anche quella psichica. In tal modo, infatti, o la sfera (della integrità) psichica esula dal concetto di salute, oppure essa, rifluendo direttamente in tale concetto, finirà con l'identificarsi con esso, ma richiando allora di ridurre la salute esclusivamente ad una condizione di soggettivo benessere psichico, quale che siano poi le condizioni fisiche della persona. In entrambi i casi, com'è evidente, si contraddirebbe la definizione di salute da cui si sono prese le mosse. 
E il rilievo della contraddizione evidenziata forse modesto sul piano argomentativo, riguardando più la paglia delle parole che non il grano delle cose si coglie con riferimento alla emersione della visione antropologica che sottostà a tali tesi: una visione, come si vedrà nel prosieguo, che contrasta nettamente col personalismo professato a parole per ricadere chiaramente o in un monismo di stampo fisicista o in un dualismo meccanicistico.

Per intanto, basti sottolineare che quando si invoca il cd. principio terapeutico o principio di totalità - per cui è lecito sacrificare un organo o una parte del corpo per la salvaguardia dell'organismo nel suo complesso - si fa rinvio ad una prospettiva che implica necessariamente sia un valore oggettivo del corpo umano e della sua integrità, sia la possibilità di instaurare una gerarchia fra gli organi e le funzioni psicofisiche. Se così non fosse, diventerebbe arbitrario scegliere di sacrificare una funzione per la salvaguardia di un'altra e sarebbe perfettamente lecito, ad es., sacrificare le funzioni cerebrali al fine di mantenere la vita vegetativa del corpo. E' ben noto, viceversa, come la stragrande maggioranza della comunità scientifica internazionale reputi inaccettabile tale strada e ricusi oggi interventi, come la lobotomia e la leucotomia, che pur non ledono tutte la funzioni cerebrali superiori ma recidono solo la sfera affettiva della persona.

Ebbene, tale principio del valore obiettivo e della gerarchia fra le funzioni viene di fatto negato laddove si afferma che la fertilità costituisce un bene o no a seconda che la persona vi abbia o no interesse, e che pertanto può essere eliminata ove lo richieda il benessere, soggettivamente inteso, della persona stessa. Ma allora, ad essere coerenti, dovremmo analogamente ritenere ammissibile, ad es., la richiesta di chi chieda che gli vengano amputate le braccia o le gambe, ove tale soggetto escluda che per lui costituiscano un bene apprezzabile.

Non si tratta di una semplice boutade: la verità è che per un verso il principio di totalità, con il correlato criterio di gerarchia fra gli organi e le funzioni, è realmente un principio indefettibile - e del resto mai seriamente contestato della medicina e dell'antropologia, e, d'altro canto, che nessuno veramente crede, in cuor suo, che la fertilità sia un bene solo se soggettivamente valutato come tale o che possa essere posto sullo stesso piano di altre funzioni, come ad es. il gusto o l'olfatto. 
In altre parole, il principio della indisponibilità dell'integrità, sia fisica che psichica, è un principio fondamentale e, al di là delle contraddittorie posizioni di comodo assunte al fine di giustificare la sterilizzazione, è la regola concreta che regge il comportamento collettivo. Basti ricordare che mentre il solo consenso non basta a rendere lecite eventuali richieste di mutilazioni o lesioni permanenti, il consenso stesso non è necessario in caso di obiettiva necessità - tanto che, contro la presunta volontà della persona, si cura pur chi abbia tentato il suicidio. Viceversa, un espresso consenso è oggi costantemente richiesto quando si tratti di realizzare una sterilizzazione non terapeutica. Accreditare una diversa impostazione in materia significherà in concreto anche questo: che in futuro $\mathrm{i}$ medici si riterranno autorizzati a procedere alla sterilizzazione eugenetica ed a quella su indicazione medica anche senza il preventivo consenso della persona.

Alla luce di tali rilievi, allora, dei diversi tipi di sterilizzazzione richiamati in apertura del discorso si giustifica soltanto quella terapeutica. Qualche dubbio permane invece per la sterilizzazione su indicazione medica, sia perché non è l'unico mezzo idoneo ad evitare gravidanze, sia perché la sua liceità potrebbe implicare anche l'ammissibilità della sterilizzazione eugenetica. Si tratta di un punto sul quale si tornerà nel prosieguo.

3. Il concetto di salute comunemente recepito, dunque, non è in grado di giustificare razionalmente la pratica della sterilizzazione antiprocreativa: essa infatti accrediterebbe una nozione talmente ampia ed elastica di salute che finirebbe col porre in crisi i fondamentali criteri concettuali ed antropologici sui quali si basa la stessa medicina.

Sembra meglio orientato, allora, il tentativo di riportare tale pratica a un diverso bene della persona: la libertà individuale, e specificamente la libertà sessuale e procreativa. In particolare, non solo la raggiunta sterilità assicurerebbe piena libertà nei rapporti sessuali, quali che siano i fini che l'individuo si ripromette, ma consentirebbe inoltre il perseguimento di scopi socialmente rilevanti o addirittura desiderabili, come la limitazione delle nascite e/o l'impedimento alla trasmissione di malattie e di caratteri ereditari indesiderati. A tal proposito anzi ma, mi sembra, giocando con la paglia delle parole - si ripropongono formule mal digerite o addirittura travisate nel loro significato 
autentico: le proposte di legge volte ad autorizzare la sterilizzazione, e presentate al Parlamento italiano, fanno ad es. espresso riferimento alla finalità di assicurare una procreazione cosciente $e$ responsabile nel quadro di un ampliamento della sfera di libertà della persona.

Ma è fin tropo facile l'obiezione che una autentica libertà in proposito non pud che chiedere il mantenimento di una effettiva, reale possibilità di scelta fra il poter procreare e il poter non procreare, mentre la sterilizzazione, con la sua concreta irreversibilità di fatto, preclude la possibilità di scelte ulteriori e diverse. Rappresenta dunque un calo, una diminuzione di libertà effettiva, tanto che qualcuno ha parlato di incosciente e irresponsabile privazione irreparabile della capacità di procreare.

La diminuzione di libertà, anzi, è talmente evidente che la diffusa condivisione di tale motivazione risulterebbe del tutto incomprensibile se non la inquadrassimo in alcune opzioni ideologiche di fondo, e cioè la sessualità come diritto assoluto, individuale ed egoista, scissa radicalmente da ogni legame con la procreazione e con il matrimonio, che infatti, in tale prospettiva, diventano solo degli ostacoli alla libera esplicazione della sessualità.

Non è possibile in questa sede un esame analitico di tutti tali punti e mi limiterò a qualche semplice richiamo. Quanto alla scissione tra sessualità e procreazione, essa è evidente nella sterilizzazione che, in quanto irreversibile, istituzionalizza il sesso come puro gioco, come ludus individuale liberatorio, teso a sfuggire il legame sociale della procreazione; ma è latente altresì nell'odierna diffusione delle pratiche di inseminazione eterologa e procreazione artificiale, irrimediabilmente divaricate da una sessualità di relazione. E si comprende allora come procreazione e matrimonio fịniscano col costituire delle realtà irrilevanti o secondarie, quando non degli ostacoli da superare per una completa libertà individuale. Basti ricordare che non è isolata nella dottrina italiana l'opinione che nega ogni rilievo al contesto familiare di chi chiede la sterilizzazione: e ciò sia per quanto concerne il requisito (suggerito de iure condendo) della già avvenuta procreazione di figli, sia per quanto riguarda il consenso del coniuge, sia infine in ordine al (denegato) rilievo della pregressa sterilizzazione, pur se taciuta al futuro coniuge, sulla validità del matrimonio (e in ciò derogando ad una chiara 
previsionc del codice civile. ${ }^{2}$ Il che, in poche parole, equivale a dire che la procreazionc non è una finalità di norma rilevante nel matrimonio, un fine che ciascuno degli sposi possa legittimamente e ordinariamente attendersi.

Si delinea in tal modo più chiaramente il quadro ideologico complessivo in cui si colloca la difesa della sterilizzazione, ma non ritengo opportuno sviluppare ulteriormente tali punti, che inevitabilmente amplierebbero il discorso ad altre tematiche. Quel che invece mi sembra necessario approfondire è se la libertà individuale rappresenti comunque una effettiva giustificazione, una ragione, più o meno solida, a difesa della facoltà di sterilizzazione.

Ebbene, anche in proposito non mancano le sorprese ove appena si approfondisca il senso e i limiti di tale invocata libertà: invero, la dottrina che non preferisce sorvolare sul punto, facendo finta di non vedere il problema, nega l'ammissibilità della sterilizzazione ove essa abbia finalità diverse da quella antiprocreativa. Si nega esplicitamente, ad es., la sua ammissibilità a scopi di sperimentazione scientifica, come parimenti, credo, dovrebbe negarsi la legittimità di una castrazione finalizzata alla fornitura di eunuchi per un harem o alla conservazione di una voce bianca. ${ }^{3}$

Si tratta peraltro di limitazioni non razionalmente giustificabili, poiché pretendono di controllare il merito delle scelte, lo scopo in vista del quale la sterilizzazione, o comunque l'intervento demolitivo, è attuato: infatti, nel momento in cui si sindaca il tipo di personalità o la direzione che ciascuno voglia dare alla propria libertà, si nega in buona sostanza che sia la libertà il valore tutelato. Si riconosce, cioè, che essa non è sufficiente a costituire il fondamento della pratica della sterilizzazione, rinviando ad un altro valore alla cui stregua avviene la valutazione di liceità o meno del comportamento.

2. L'art. 122 c.c. dispone che il matrimonio può essere annullato su istanza del coniuge caduto in errore su "qualità personali ed essenziali dell'altro coniuge" qualora l'errore riguardi, fra l'altro, "l'esistenza di una malattia fisica o psichica o di una anomalia o deviazione sessuale tali da impedire lo svolgimento della vita coniugale". E dunque se, in tale interpretazione, la sterilizzazione volontaria non costituisce neanche anomalia tale da impedire un ordinario svolgimento della vita coniugale, se ne deve dedurre che la procreazione non può dirsi attinente nonché ad un fine essenziale del matrimonio, neppure ad un naturale negotii, a un fine che ciascuno dei coniugi può legittimamente e ordinariamente attendersi dal matrimonio stesso.

3. Si tratta di ipotesi, già largamente sperimentate nella storia umana, oggi improbabili a verificarsi per il divario fra la pubertà e la maggiore età richiesta per una scelta volontaria in tal senso, ma non impossibili in assoluto. 
S'è già veduto, peraltro, che tale valore non può essere la salutc, che implica una irrinunciabile valenza obiettiva, a pena di togliere legittimità a qualsiasi tipo di cura e intervento medico-chirurgico. Rimane allora, come possibile fondamento di tale libertà, un altro bene: in concreto, e nella valutazione dei fautori della sterilizzazione, il finc sociale della limitazione delle nascite, su cui è necessario ora soffermare l'attenzione.

4. Il tema della limitazione delle nascite, come problema sociale legato alla sovrappopolazione, è troppo noto perché sia necessario soffermarsi sui suoi termini generali.

Piuttosto, è il caso di sottolineare come al fondo di tali concezioni sta una ormai non più latente impostazione che, se ancora è disposta a vedere nella procreazione non limitata un bene individuale, a condizione che l'interessato lo consideri tale, vede tuttavia in essa, senza mezzi termini, un male sociale. Sempre più massicciamente infatti si prospetta un pressante interesse collettivo a limitare il numero delle nascite, sì che appare benvenuta ogni pratica che consenta di favorire tale finalità, sia essa l'adozione di anticoncezionali, la sterilizzazione e, come ultima risorsa, lo stesso aborto. E ciò, si badi, non solo nei paesi caratterizzati da alti tassi di crescita demografica, ma anche laddove, come in Italia e in molti altri paesi, si è arrivati al declino demografico, alla cd. crescita negativa.

Quanto alle giustificazioni di tale opzione, esse sono ovviamente diverse per le diverse realtà socio-economiche: nei paesi in via di sviluppo, una drastica riduzione del numero delle nascite viene ritenuta condizione imprescindibile dello sviluppo economico; nel mondo industrializzato la si ritiene piuttosto necessitata da esigenze di equilibrio ecologico, dall'esigenza di ridurre la pressione umana sulla natura e il prelievo di risorse.

Possiano tranquillamente lasciare da parte affermazioni polemiche, come quella che protesta contro una presunta politica pronatalista dell'occidente, finalizzata al mantenimento di un alto tasso di incremento demografico a sua volta funzionale al sottosviluppo economico del terzo mondo. Si tratta di affermazioni risibili e in palese contrasto con una prassi politica mondiale che dagli anni ' 60 in poi ha imposto ai paesi emergenti, quale condizione 
irrinunciabile per l'aiuto economico, una radicale politica di limitazione delle nascite. Ed c̀ ben noto, poi, come su questa strada si siano particolarmente distinti alcuni paesi asiatici, come l'India e la Cina, dove è stata anzi sistematicamente praticala, con alterne fortune, la sterilizzazione forzata di larghi strati della popolazione.

Quel che è certo è comunque una cosa: anzitutto mancano seri studi economici che supportino in modo scientificamente corretto l'asserità incompatibilità tra incremento demografico e sviluppo economico. Essa cioè costituisce allo stato attuale una affermazione gratuita, dedotta empiricamente dalla constatazione che paesi con alti tassi di crescita demografica hanno bassi livelli di sviluppo economico, ma non è stata mai seriamente dimostrato un rapporto di causa-effetto tra i due fenomeni.

Ma soprattutto, studiosi della levatura di un Maynard Keynes sostengono proprio il contrario, rilevando che lo straordinario sviluppo economico e tecnologico dell'occidente negli ultimi due secoli è legato ad un parimenti eccezionale livello di incremento demografico. Non è un caso del resto se gli Stati Uniti d'America hanno da alcuni anni cambiato rotta e tolto il loro appoggio economico a politiche che pratichino l'aborto e la sterilizzazione coatta come strumenti per il controllo delle nascite, imputando piuttosto la stagnazione economica di tali paesi a distorte politiche di pianificazione dell'economia e collettivizzazione delle risorse.

E' sotto gli occhi di tutti, poi, che molti dei paesi sottosviluppati devono tale loro condizione ad una politica di potenza, magari soltanto regionale, che destina enormi risorse agli armamenti piuttosto che alla creazione delle infrastrutture economiche necessarie allo sviluppo. Del resto, le colpe non stanno soltanto dalla loro parte e molte distorsioni vanno imputate alla politica cieca di diversi paesi occidentali che, sotto l'etichetta di crediti agevolati allo sviluppo, alimentano una politica di consumismo - leggi: di acquisto di prodotti occidentali che distrugge risorse che dovrebberro essere destinate alle infrastrutture di sostegno al decollo economico.

$\mathrm{Si}$ aggiunga che quello della sovrappopolazione è un problema non solo limitato ad alcune zone del globo, ma anche contingente, di breve periodo, e appare quanto meno azzardato tentare di risolverlo generalizzando una scelta così drastica e definitiva. Non si dica che in una situazione in cui l'aborto è 
largamente praticato la sterilizzazione costiluirebbe un male minore: un male può essere soltanto subito, non scelto volontariamente come risposta ad altri mali. Se non altro perché l'esperienza dimostra che la prassi abortista ha potuto prosperare proprio innestandosi su una mentalità che potremmo definire antinatalista a tutti i costi, su una attitudine mentale cioè che, a dir poco, si illude di risolvere i problemi scegliendo delle scorciatoie, non importa se irrispettose della dignità umana.

Certo, nessuno vuol negare che l'equilibrio fra le risorse e la popolazione è un problema importante, che non può essere semplicemente ignorato, ma di esso, appunto, devono considerarsi tutti gli aspetti, mantenendo viva la consapevolezza che le risposte ad esso devono essere autenticamente rispettose della libertà e della dignità dell'uomo. Lasciato allora all'economista e al politico il loro compito, al giurista compete la domanda se la sterilizzazione antiprocreativa sia comunque una risposta accettabile dal punto di vista relativo al rispetto dei diritti dell'uomo.

5. Sotto tale profilo, la risposta non può che essere negativa. Fermissima la condanna di ogni forma di sterilizzazione forzata o anche soltanto incoraggiata dallo Stato, perché contraria alla dignità e alla libertà della persona, altrettanto fermo dev'essere il rifiuto di quella volontaria.

Diversi possono essere, al riguardo, gli approcci al tema. Ne scelgo uno che in qualche modo può dirsi comune alle esperienze italiana a brasiliana, in parte sul piano del diritto positivo, in parte sul terreno di quei principi generali del diritto che, se pur faticano oggi a trovare un univoco fondamento, convergono tuttavia, nell'universale apprezzamento, sulla esigenza di tutela della libertà e dignità della persona umana.

Quanto allo spunto di diritto positivo, l'art. 5 del codice civile italiano vieta gli atti di disposizione del proprio corpo quando cagionino una diminuzione permanente della integrità fisica o siano altrimenti contrari ... all'ordine pubblico o al buon costume. Il richiamo a tale norma si giustifica sia perché, caduta la repressione penale della sterilizzazione volontaria, la dottrina italiana fonda proprio su tale disposizione il divieto di tale pratica, sia perché alcuni dei progetti di riforma del codice civile brasiliano contengono proprio una disposizione di 
analogo tenore. Inoltre, ben può dirsi che tali previsioni sulla indisponibilità del corpo, nel loro contenuto sostanziale, fanno comunque parte del patrimonio culturale della nostra civiltà occidentale, di là dalla loro espressa formulazione in testi legge, si che si prestano ad essere assunte come comune punto di riferimento.

Ebbene, tali principi si spiegano solo alla luce di una antropologia che potremmo sintetizzare qui col termine personalismo, intendendo con tale termine una visione globale della persona umana che la vede a pari titolo costituita sia dal corpo che dallo spirito. In tale visione, cioè, il corpo è per così dire altrettanto umano dell'intelletto; non è, per intenderci, quella gabbia in cui Platone vedeva rinchiusa l'anima, quel carcere da cui l'anima deve liberarsi per essere autenticamente spirituale, e perciò veramente umana. E' questo il cd. dualismo che, originatosi nella cultura greca, ha influenzato non poco la stessa cultura occidentale e cristiana se un pensatore come Cartesio potè configurare il corpo come mera macchina, aliena e sostanzialmente estranea all'anima (e sia pure intesa quest'ultima come sinonimo di coscienza).

L'impostazione che si è designata sinteticamente come dualismo opera ancor oggi potentemente nella visione di tanti pensatori contemporanei: basti qui ricordare, come segno della scissione tra lo spirito e il sostrato fisico del corpo, alcuni dei cd. nuovi filosofi (e in particolare Baudrillard e Lyotard, Deleuze e Guattari) e la loro allucinata visione del mondo come un universo di desiderio, dove gli uomini sono ridotti niente più che a semplici macchine desideranti. Una visione, certo, cui in qualche modo offrono oggi suggestioni anche le moderne tecniche medico-chirurgiche e le pratiche dei trapianti d'organo che, quasi moderni pezzi di ricambio, illudono ciascuno di noi di potersi in perpetuo rinnovare, d'essere altro che un corpo e uno spirito inscindibilmente contessuti insieme.

Ciò che può apparire singolare, allora, è che in tale impostazione per così dire assolutamente libertaria l'uomo non è il corpo. Al contrario, la scissione tra corporeità, sessualità e procreazione rese dalle moderne tecniche biomediche progressivamente autonome dai vincoli biologici e non più interdipendenti - ha finito per fare dell'uomo quasi solo uno spirito, rectius: una pura volontà desiderante che, aspirando ad affrancarsi dai limiti biologici del corpo, finisce col perdere la propria identità e perciò, già nella visione di tale 
filosofi, muore come soggetto: non solo sul piano epistemologico (come avveniva con Foucault), ma ormai, con Deleuze e Guattari, anche sul piano ontologico. Qui dunque, come ben è stato detto, la metafisica della possibilità, filosofica e tecnologica, diviene metafisica della soggettività e vi si celebra l'esplosione, il trionfo del Desiderio come nuova categoria trascendentalc assoluta. Non esiste più l'uomo: c'è solo un universo di desiderio che opera ciccamente e pensa sc stesso nel deserto delle sue macchine desideranti.

$E^{\prime}$ culturalmente in declino, invece, ma d'altra parte operativamente ancora vigorosa nel comportamento di tanti l'altra opzione che in questo secolo $\mathrm{e}$ in quello passato ha conteso il campo alle impostazioni sopra ricordate: quella del cd. monismo che ha trovato in Marx, Sartre e Marcuse i suoi più noti, pur se disomogenei, propugnatori. $\mathrm{E}^{\prime}$ la visione dell uomo a una dimensione, in cui il corpo esaurisce la totalità della persona e delle sue esperienze. E' il corpo come luogo e mezzo di liberazione, di piacere, di gioco, che va leeciberato dalla legge e dalla morale, dalle sovrastrutture e dai vincoli sociali: in una parola, da ciò che definiamo spirito o ragione umana.

6. 11 personalismo, viceversa, ci ricorda che l'uomo è inscindibilmente corpo e anima, carne e spirito. L'uomo cioè non ha un corpo, così come non ha un sesso: l'uomo, piuttosto, è anche un corpo così come è anche, inseparabilmente, spirito. $\mathrm{E}$ in tale inscindibile unione l'uomo sarà poi, parallelamente, maschio o femmina. Lo spirito, dunque, compagina il corpo, ne è la forma sostanziale, e insieme costituiscono l'uomo nella sua unità: quel che si definisce, appunto, persona umana.

Solo in questa visione, del resto, si percepisce e comprende la rinnovata attenzione moderna ai diritti del corpo, alle istanze e necessità della vita materiale, che tanto potentemente hanno contribuito a porre in crisi regimi politici e sociali dimantichi di questa elementare verità. Solo così si comprende come ogni aggressione o lesione del corpo sia vissuta e sofferta come una lesione della persona tutta intera, e come, infine, la dignità della persona reclami anche il rispetto del suo corpo, se non altro perché è solo tramite il corpo che l'uomo entra nel mondo e in contatto con gli altri. Senza il corpo, cioè, mancherebbe lo stesso essere con gli altri, quel che Scheler definisce l'epifania dell'io. 
E ancora, solo in tale prospettiva ha senso quel principio di totalità che tutti alfermano di condividerce e in virtù del quale è poi possibile e giustificata l'altivilà medica e chirurgica. Solo in tal modo, cioè, si comprende sia la riconduzione di entrambe le dimensioni, fisica e psichica, nel concetto di salute globale della persona, sia la imprescindibile gerarchia che va instaurata fra le parti c il tutto nonché fra le varie funzioni della persona.

Così, sessualità e procreazione sono una dimensione essenziale dell'uomo, c la facoltà di procreare, in particolare, non è facoltà che possa essere assimilata ad es. all'olfatto. Affermare il contrario; o legare la valutazione di essenzialità all'apprezzamento del singolo, equivarrebbe a negare l'evidenza, sia per la valutazione 'diffusa già nel corpo sociale, sia per il diverso rilievo che una menomazione di tali facoltà avrebbe, ad es., sul piano risarcitorio.

Conseguentemente, se il principio di inviolabilità della persona ricomprende necessariamente, per comune riconoscimento, anche l'indisponibilità da parte dello stesso soggetto, deve riconoscersi che la facoltà di procreare non è dimensione di cui si possa disporre al di fuori degli stessi limiti in cui è possibile disporre di altre essenziali facoltà o attitudini.

In buona sostanza, il concetto di salute non può essere ridotto a una nozione bon a tout faire, a un concetto elastico o di comodo, variabile in relazione alle circostanze, idoneo a giustificare qualsiasi compromissione dell'integrità. Più specificamente, la generica nozione di benessere psichico non può giustificare la soppressione della facoltà di procreare. Sotto tale più ampio profilo, perciò, si conferma come lecita la sola sterilizzazione terapeutica, quella sterilizzazione cioè che, in presenza di un pericolo serio e attuale per la vita della persona, appaia necessaria per la salvezza dell'organismo nel suo complesso.

7. Con riguardo alla sterilizzazione su indicazione medica, il discorso è più delicato, mancando quell'estremo della attualità del pericolo che costituisce necessario requisito per la scriminante dello stato di necessità. ${ }^{4}$

Ora, con riguardo almeno all'ordinamento giuridico italiano, essa potrebbe forse ritenersi lecita quando ricorrano gli estremi di un pericolo grave e medicalmente accertato per la vita della madre. Non ci si può nascondere, però,

4. Così dispongono sia l'art. 2045 del codice civile, sia l'art. 54 del codice penale italiani. 
che tale ammissibilità richiederebbe l'elaborazione di un autonomo principio, desunto in via interpretativa dalla unione di regole diverse: la scriminante dello stato di necessità, per la cui piana applicazione manca appunto il requisito della attualità del pericolo, e la regola, enunciata dalla Corte costituzionale, sulla liceità della interruzione di gravidanza in caso di pericolo grave e medicalmente accertato per la vita della madre (sent. n. 27/1975). ${ }^{5}$

Si tratterebbe, allora, di operare una integrazione del sistema in via interpretativa che appare teoricamente non impossibile - pur se mancano concrete proposte in tal senso - e che mi limito a prospettare come una delle soluzioni praticabili alla luce dell'ordinamento positivo italiano. Essa peraltro, e come vedremo, dà ingresso ad una impostazione nella quale si indebolisce il divieto di altri tipi di sterilizzazione.

8. Va esclusa in ogni caso, viceversa, la liceità della cd. sterilizzazione eugenetica. Certo, in tali ipotesi la richiesta dell'interessato non è sicuramente capricciosa, non costituisce cioè una sterilizzazione edonistica o di comodo.

E tuttavia il problema è che, una volta travalicati certi limiti di rispetto dell'ordine naturale, non si riesce a trovare una coerente linea di confine sulla quale arrestarsi. Che dire infatti dei casi in cui una malata di mente, per la peculiare affezione, sia esposta a un uso sconsiderato della sessualità e soggette dunque a subire gravidanze a ripetizione? Certo non potremo e non dovremo affidarci ad una sua richiesta. Ricorreremo allora alla sterilizzazione coatta? Ma cos'altro ha fatto il nazismo, negli anni immediatamente precedenti la seconda guerra mondiale, se non una politica eugenetica per i malati di mente e le persone tarate? E quale sarà il limite di fronte al quale ci fermeremo? Come potremo ciò razionalmente e sicuramente distinguere un semplice disturbo della personalità da una vera e propria malattia mentale che escluda ogni discretionem iudicii?

E soprattutto, con quale criterio opereremo una sterilizzazione coatta dei malati di mente e la escluderemo per i portatori di tare e malattie ereditarie?

5. E' ben vero che la successiva legge del 1978 , che ha disciplinato l'interruzione volontaria della gravidanza, ha ampiamente valicato tali limiti, ma essa, proprio per tale motivo, viene ritenuta costituzionalmente illegittima da non pochi settori della dottrina e ritengo perciò inaffidabile la strada di dedurne principi e regole da applicare ad altre problematiche. 
Se ci preoccupiamo cioc̀ della salute dei malati di mente, in quanto non in grado di provvedere a se stessi, come potremo non occuparci poi di creature, anch'esse per intanto incapaci di far sentire la propria voce, che verranno al mondo affette da gravi tare ereditarie? Ed affermato così il principio che la vita non è degna di essere vissuta se affetta da particolari malattie, non scivoleremo fatalmente in quella stessa ottica che ha consentito al nazismo l'eliminazione sistematica e pianificata delle $\mathrm{cd}$. Lebensunwertenlebens? (e cioè, esistenze prive di valore vitale: espressione che, nel linguaggio odierno, suona proprio vite non degne di essere vissute). Non è un caso del resto se in Europa la dottrina più tenacemente contraria ad ogni forma di sterilizzazione è proprio quella tedesca, ammaestrata in tal senso dalla tragica esperienza del nazismo.

In altre parole, la sterilizzazione eugenetica volontaria apre fatalmente la strada alla sterilizzazione coatta, sia dei malati di mente sia dei portatori di tare ereditarie e va pertanto decisamente respinta come contraria alla dignità della persona.

9. A voler trarre le fila di quanto s'è detto, allora, va negata l'ammissibilità della sterilizzazione, come pratica diretta al controllo della natalità, sia forzata che volontaria, edonistica o eugenetica che sia (cd. sterilizzazione antiprocreativa). Giustificata, nel complessivo quadro di riferimento, appare la sterilizzazione terapeutica e problematica, pur se forse ammissibile con riguardo all'ordinamento positivo italiano, quella su indicazione medica.

A tale conclusione, come s'è cercato di evidenziare, si giunge attraverso l'applicazione di principi e regole giuridiche che ben possono dirsi generali, in quanto comuni alla civiltà giuridica occidentale. Essi cioè appaiono fondati su quel rispetto della libertà e della dignità della persona umana che, per comune riconoscimento, costituiscono ormai il fondamento e il fine di ogni ordinamento giuridico, e per le quali non sono del resto mancate, negli anni, le riaffermazioni solenni: dalla Dichiarazione universale dei diritti dell'uomo fino alla più recente Dichiarazione di Helsinky.

10. Più delicato è invece, per concludere, il discorso dei modi e criteri con cui tali principi possono o devono essere recepiti dagli ordinamenti positivi dei 
singoli Stati. Pur esclusa radicalmente, infatti, la legitlimità di qualsiasi forma di sterilizzazione forzata, le singole legislazioni prospettano un ventaglio di diverse scelte politico-legislative, da quella che favorisce la sterilizzazione volontaria, assumendola come servizio pubblico nel sistema sanitario nazionale, fino a quella che la vieta presidiando il divieto con sanzioni penali.

La scelta di fare della sterilizzazione un servizio pubblico sanitario mi sembra nettamente da respingere - oltre che sul piano giuridico, come s'è visto anche sul piano sociopolitico, come quella che crea un alibi ai governi, fornisce una soluzione di comodo ai problemi e allontana l'adozione di adeguate politiche sociali di sostegno alle famiglie e di sviluppo economico. Essa poi è di fatto destinata ai ceti sociali più deboli e mostra di ignorare che un adeguato sviluppo economico risolve da solo tali problemi, come dimostra l'esperienza dei paesi industrializzati, ormai alle prese con problemi di grave declino demografico.

Quanto alla scelta di una repressione penale della sterilizzazione volontaria, essa non può che dipendere dal criterio generale del bene comune: solo se esso lo richieda potrà giungersi a tanto. Nel nostro caso, non essendo in gioco la violazione grave di diritti altrui, ben può lo Stato rinunciare a tale alternativa, del resto abbandonata ormai, a quanto mi risulta, su scala mondiale.

Non può invece, lo Stato, rinunciare ad additare la via di un integrale rispetto della persona, poiché l'ordinamento giuridico ha anche irrinunciabili compiti di indirizzo del corpo sociale e dei comportamenti collettivi. E dunque mi sembra pienamente giustificata una soluzione, sicuramente ammissibile ad es. per l'ordinamento italiano, che veda nella sterilizzazione volontaria antiprocreativa un comportamento illecito, se pure tollerato dallo Stato al pari di altre realtà sociali. 6

In concreto, perciò, i singoli avranno una facoltà di mero fatto di sottoporsi alla sterilizzazione volontaria, ma il contratto per l'operazione chirurgica, essendo diretto a una menomazione permanente e grave dell'integrittà della persona non giustificata da una necessità di salvaguardia della sua salute, avrà un oggetto illecito e sarà perciò nullo.

6. Ad es., nell'ordinamento italiano il gioco e la scommessa sono leciti in alcuni casi, tollerati in altri, illeciti e radicalmente vietati, infine, in taluni casi particolari: v. artt. $1933 \mathrm{ss}$. 
Le conseguenze, come si comprende, sono di non poco rilievo. Sul piano civilislico, ad es., non si darà azione per il pagamento del prezzo dell'operazione chirurgica, pur se non sarà ammessa l'azione di ripetizione, trattandosi di prestazione contraria al buon costume. Inoltre, l'eventuale, mancato conseguimento del risultato - lo stato di infertilità della persona non darà luogo ad azione di risarcimento, neppure nel caso in cui segua poi una procreazione. Daranno diritto al risarcimento invece eventuali lesioni personali conseguenti all'intervento, ma in base alle sole norme sulla responsabilità extracontrattuale, $\mathrm{e}$ competerà perciò al danneggiato, fra l'altro, provare la negligenza del medico.

La sterilizzazione volontaria, inoltre, costituirà causa di nullità del matrimonio sotto il profilo di un errore essenziale nelle qualità della persona o, se successiva al matrimonio stesso, causa di separazione personale con addebito. $^{7}$

Quanto ai profili pubblicistici, ne consegue che la sterilizzazione volontaria antiprocreativa non potrà essere eseguita nelle strutture pubbliche, non attuando l'interesse collettivo alla salute delle persone, e la stessa sterilizzazione su indicazione medica, per il caso di pericolo per la salute della madre, potrà essere rifiutata da singoli medici che oppongano il contrasto di essa con le proprie convinzioni morali: è la cd. obiezione di coscienza che ha trovato in Italia ampio riconoscimento per il caso dell'aborto. Va aggiunto anzi che l'obiezione di coscienza potrà essere sollevata, per tutti i casi di sterilizzazione non terapeutica, anche dai medici che operino in cliniche mediche private, senza che ciò costituisca inadempimento del contratto che li lega alla struttura sanitaria.

* Conferência proferida na Faculdade de Direito da USP em 10 de setembro de 1991.

7. Cfr., per l'ordinamento italiano, l'art. 122 c.c., citato sopra, e l'art. 151 , per il quale "la separazione può essere chiesta quando si verificano ...fatti tali da rendere intollerabile la prosecuzione della convivenza o da recare grave pregiudizio alla educazione della prole" " $l$ giudice, pronunciando la separazione, ...dichiara a quale dei coniugi sia addebitabile la separazione, in considerazione del suo comportamento contrario ai doveri che derivano dal matrimonio". 